\title{
The tumor suppressor DAP-kinase links cell adhesion and cytoskeleton reorganization to cell death regulation
}

\author{
Ruey-Hwa Chen*, Won-Jing Wang \& Jean-Cheng Kuo \\ Institute of Molecular Medicine, College of Medicine, National Taiwan University, Taipei, Taiwan
}

(C) 2006 National Science Council, Taipei

Key words: anoikis, cell death, cytoskeleton, DAP-kinase, tumor suppressor

\begin{abstract}
Summary
Death-associated protein (DAP)-kinase, an actin-cytoskeleton localized serine/threonine kinase, functions as a novel tumor suppressor and participates in a wide variety of cell death systems. Recent studies indicate that DAP-kinase elicits a potent cytoskeletal reorganization effect and is capable of modulating integrin inside-out signaling. Using this understanding of DAP-kinase protein function as a framework, we discuss the functional mechanisms of this kinase in regulating death-associated morphological and signaling events. Furthermore, a potential role of DAP-kinase to be a drug target is also discussed.
\end{abstract}

\section{Introduction}

Death-associated protein (DAP)-kinase is a member of a novel family of serine/threonine kinases. The members of DAPK family, including DAPkinase, DRP-1/DAPK2, Dlk/ZIP kinase, DRAK1 and DRAK2, share highly conserved catalytic domains but differ in their extra-catalytic domains $[1,2]$. These structure features account for their phosphorylation of common substrate and capability of inducing apoptosis and yet diverse subcellular localization and certain unique activities. DAP-kinase is considered as the prototype of this family, as it is the first family member to be identified and has been most intensively studied. DAP-kinase was discovered through a genetic screen in which an anti-sense library was used to identify genes involved in interferon- $\gamma$-induced cell death in HeLa cells [3]. This $160-\mathrm{kDa}$ multidomain protein contains not only a kinase domain and calmodulin binding motif essential for its catalytic activity, but also domains for protein-protein

*To whom correspondence should be addressed. Fax: + 886-223957801; E-mail: rhchen@ha.mc.ntu.edu.tw interactions, such as the ankyrin repeat domain and death domain. In addition, a segment adjacent to ankyrin repeats is required for the cytoskeleton targeting of this kinase [3, 4]. Similar to other calmodulin-dependent kinases, the catalytic activity of DAP-kinase is primarily regulated by binding of calcium-activated calmodulin to the calmodulin binding motif [4], which likely leads to a change in conformation thereby exposing the active site. In addition, the catalytic activity of DAP-kinase can be fine-tuned by posttranslational modifications. DAP-kinase was demonstrated to undergo autophosphorylation at Ser308 which lies in the calmodulin binding motif. This phosphorylation reduces the affinity for the calmodulin and thus attenuates DAP-kinase catalytic activity [5]. This negative regulation of DAP-kinase activity via autophosphorylation is a unique feature of certain DAPK family kinases. This mode of regulation allows the activity of DAP-kinase to be in-checked in healthy cells, as full activation of DAP-kinase would require the combination of two signals, i.e., the activation of an unidentified phosphatase to dephosphorylate Ser308 and the increase of intracellular calcium. In addition to 
Ser308, DAP-kinase can also be phosphorylated at Ser 735 by ERK, which leads to an upregulation of DAP-kinase catalytic activity when tested both in vitro and in vivo. Kinetic analysis indicates that this phosphorylation decreases the $\mathrm{Km}$ value of DAP-kinase, thus increasing the binding affinity for DAP-kinase substrates [6]. As Ser735 resides in the cytoskeleton-binding region, this finding raises a possibility that the cytoskeleton-binding region may be positioned in proximity to the catalytic pocket in the 3-D structure of DAP-kinase.

\section{DAP-kinase as a tumor suppressor}

In mice and human, DAP-kinase mRNA was found to be ubiquitously expressed in various tissues, with the highest expression level observed in lung and brain [7]. Expression of DAP-kinase mRNA or protein, however, is frequently lost in certain human cancer cell lines and biopsies derived from human tumors (for review see [7, $8]$ ). In most cases, this loss of DAP-kinase expression is resulted from hypermethylation-triggered gene silencing, and a $\mathrm{CpG}$ island at the $5^{\prime} \mathrm{UTR}$ of DAP-kinase gene was found to be a target of hypermethylation [9]. To date, a large number of studies indicate that DAP-kinase gene is abnormally hypermethylated in certain preneoplastic lesions and a variety of tumor tissues of different origins, such as B cell lymphoma [9, 10], T- and NK-cell malignancies [11], nasopharyngeal carcinoma [12, 13], non-small cell lung carcinoma [14], head and neck cancer $[15,16]$, colon carcinoma $[17,18]$, gastric cancer [17, 19], and hepatocellular carcinoma [20]..., etc. Furthermore, in many circumstances, DAP-kinase hypermethylation associates with advanced tumor grades/stages, increased tumor recurrence, and/or decreased survival rate $[10,14,16,20-27]$, and therefore DAP-kinase hypermethylation is considered as a diagnostic/prognostic marker for several types of cancers. Collectively, these findings suggest that silencing of DAP-kinase confers a selective advantage of cancer development and progression.

Consistent with the notion that loss of DAP-kinase plays a causative role in tumor development, the anti-tumorigenic function of DAP-kinase was directly demonstrated in a mouse model system [28]. In this Lewis lung carcinoma system, the expression level of DAP-kinase is inversely correlated with the metastatic activities of the tumors and restoration of DAP-kinase expression in the highly metastatic Lewis lung carcinoma to physiological level efficiently suppresses their ability to form metastases in mice. The tumor suppression activity of DAP-kinase has also been demonstrated in the in vitro cell culture system. It was found that DAP-kinase is capable of suppressing c-myc- and E2F-induced oncogenic transformation in a p53-dependent manner [29]. A detail molecular dissection indicates that DAPkinase increases the p53 protein level and p53responsible genes and participates in the oncogeneinduced, p53-dependent apoptotic checkpoint pathway. Thus, these studies suggest that DAPkinase suppresses tumorigenesis through at least two mechanisms, i.e., linking apoptosis to antimetastasis and channeling oncogene activation to p53-dependent apoptotic checkpoint.

\section{DAP-kinase in cell death}

Ever since the initial identification of DAP-kinase involvement in the interferon- $\gamma$-induced cell death, the role of this kinase in various cell death systems has been a subject of intense investigation. To date, DAP-kinase has been found to participate in a wide variety of death systems, and therefore is considered to be a general death mediator. For instance, inhibition of DAP-kinase by dominantnegative mutant, anti-sense RNA or overexpression of its death domain suppresses apoptosis triggered by several cytokines, such as TGF- $\beta$ [30], Fas ligand, TNF- $\alpha$ [31] and TRAIL [32]. Dissection of the functional position of DAP-kinase in the apoptotic pathway of TGF- $\beta$ indicates that DAP-kinase acts downstream of Smad and upstream of mitochondrial-based pro-apoptotic events [30]. Consistent with the notion that DAP-kinase acts upstream of mitochondrial death events, Bcl-2 has been shown to protect apoptotic death induced by the active mutant of DAP-kinase [31]. A possible effect of DAP-kinase on mitochondria, either directly or indirectly, may account for its broad involvement in various apoptotic systems.

One of the important pathological effects of DAP-kinase lies in its participation in cell death of the central nervous system. The expression of DAP-kinase is particularly enriched in brain and 
displays a developmentally regulated manner [33]. A number of studies revealed that DAP-kinase not only mediates neuronal death triggered by various death stimuli and pathological conditions, but also is upregulated at the activity or expression level in response to such death stimuli. For instance, DAPkinase has been shown to play a role in neuronal cell death induced by C2-, C6-, and C8-ceramides $[34,35]$, seizure [36], and various ischemia conditions [37, 38]. In vivo, retinal ganglion cells for DAP-kinase null mice displays increased survival following the treatment of cytotoxic level of glutamate [33]. As all of these findings point out a possible role of DAP-kinase in neurodegenerative disorders and ischemic neuronal injury, DAPkinase is now considered as an attractive target for treatment of CNS injury and degenerative diseases. An aminopyridazine-based selective inhibitor of DAP-kinase has been developed recently and this small molecule displays neuroprotective effect in both hypoxia- and ischemia-induced brain injury models [37, 38]. In addition to being involved in the pathological death conditions in neurons, recent study also suggests a function of DAP-kinase in developmentally regulated neuronal cell death. The netrin receptor $\mathrm{UNC} 5 \mathrm{H}$ has recently been demonstrated as a dependence receptor, because it triggers apoptotic cell death in the absence of ligand, netrin-1 [39]. Intriguingly, DAP-kinase was found to be recruited to UNC5H2 partly through the interaction between their death domains. In the absence netrin-1, UNC5H2 reduces DAP-kinase autophosphorylation at Ser308 and thus increases DAP-kinase catalytic activity. Furthermore, dominant-negative inhibition of DAP-kinase partially impairs UNC5H2-triggered apoptosis [40]. Therefore, DAP-kinase may function in the cell fate determination during neuronal development.

In addition to apoptotic cell death, DAP-kinase can also induce autophagic death in certain cell systems. Autophagy is a process in which intracellular membrane structures sequester proteins and organelles to degrade and turn over these materials. Autophagy is induced when cells encounter environmental stresses, such as nutrient starvation and pathogen infection and results in either adaptation and survival or cell death [41, 42]. The autophagic cell death (or type II cell death) is biochemically and morphologically distinct from apoptosis (or type I cell death). In autophagic death, caspase activation and DNA fragmentation do not occur. Instead, autophagic death is characterized by the appearance of double- or multiple-membrane cytoplasmic vesicles engulfing bulk cytoplasm and/or cytoplasmic organelles, such as mitochondria and Golgi [41, 42]. DAP-kinase induces autophagic death in MCF7 and HeLa cells and is necessary for interferon- $\gamma$-induced autophagosome formation in HeLa cells [43]. It remains to be investigated how cells choose to undergo different death modes in response to DAP-kinase activation. As autophagic death can be activated when apoptotic effectors, such as caspases [44] or Bax/Bak [45], are inhibited, it wound be intriguing to determine whether cells switch between DAP-kinase triggered autophagic death and apoptosis by the availability of different death effectors and/or DAP-kinase substrates.

\section{DAP-kinase in regulating actin cytoskeleton}

Biochemical and immunostaining analyses indicate that DAP-kinase is localized to the cytoskeleton in association with the actin microfilament system and this localization requires both the ankyrin repeat and cytoskeleton binding domains of DAP-kinase. Deletion of the cytoskeleton binding domain leads to a diffuse cytoplasmic localization, whereas a mutant lacking ankyrin repeat domain mislocalizes to focal adhesions [46]. As the kinase domain of DAP-kinase is related to that of myosin light chain kinase (MLCK), myosin-II regulatory light chain (MLC) becomes a potential in vivo substrate of DAP-kinase. Indeed, both biochemical and immunostaining analyses revealed that DAP-kinase phosphorylates MLC at Ser19 in vivo [46, 47]. Through this phosphorylation event, DAP-kinase promotes the contractility of actomyosin system and is capable of stabilizing stress fibers in serum-starved conditions. Furthermore, DAP-kinase activity is involved in serumstimulated stress fiber formation in fibroblasts [47]. Consistent with its ability to phosphorylate MLC, DAP-kinase overexpression in HeLa cells induces a dramatic change in cell morphology, including the formation of protrusions and membrane blebbing [46]. Thus, DAP-kinase-induced MLC phosphorylation is considered to contribute as least in part to the cytoskeleton changes observed in 
apoptotic cells. In contrast to other kinases that phosphorylate MLC, DAP-kinase is incapable of stimulating focal adhesion assembly in serum-starved conditions, and even causes focal adhesion disruption in serum-stimulated conditions [47]. This uncoordinated regulation of stress fibers and focal adhesions is a unique feature for DAP-kinase and might eventually lead to cell detachment and anoikis (see below). Since phosphorylation of MLC alone would promote, rather than inhibit focal adhesion assembly, these findings suggest the existence of unidentified DAPkinase substrate that is responsible for negatively regulating focal adhesions.

\section{DAP-kinase as an anoikis inducer by inactivation of integrin}

Anoikis is defined as a subset of apoptosis that is triggered by loss of or inadequate cell-matrix interaction [48]. Adhesion to extracellular matrix regulates cell survival through both integrin engagement and appropriate cell spreading. Resistance to anoikis is expected to confer a selective advantage on precancerous epithelial cells, affording them an increased survival time in the absence of matrix attachment, and facilitating eventual reattachment and colonization of secondary sites [48]. Indeed, breakdown of anoikis has been reported to contribute prominently to the malignancy of mammary, lung and colon cancers [4951]. Recent study revealed that DAP-kinase suppresses integrin-mediated cell adhesion through an inside-out inactivation of $\beta 1$ integrin. Furthermore, enforced activation of integrin by functional activating antibody abrogates the apoptotic effect of DAP-kinase. These findings indicate that DAPkinase induces apoptosis through inhibition of integrin and subsequently blockage of matrix survival signal, and thus identify DAP-kinase as a novel anoikis inducer. Consistent with this notion, DAP-kinase induces apoptosis in anoikis-sensitive epithelial cells, but not in anoikis-resistant carcinoma cells [52]. Importantly, we and other groups found that blockage cell adhesion and integrin survival signals leads to the induction of p53 [52-54], which may explain the role of p53 in cell susceptibility to DAP-kinase triggered apoptosis. Notably, p53 disrupts mitochondrial membrane potential and permeability either directly or indirectly through transcriptional activation of several Bcl-2 family proteins [55-57]. Furthermore, loss of cell-matrix interaction has been reported to affect multiple $\mathrm{Bcl}-2$ family proteins at different levels, such as transcription, posttranslational modification and subcellular localization, which ultimately lead to mitochondrial-dependent apoptosis (for review see [58]). In these regards, the function of DAP-kinase as an anoikis inducer correlates well with the finding that DAP-kinase acts upstream of mitochondrialdependent apoptotic events.

The ability of DAP-kinase to suppress integrin activation places DAP-kinase to the growing list of intracellular molecules that regulate integrin inside-out signaling. These molecules control integrin activation through their action on integrin cytoplasmic domains, which induce conformational changes in the integrin extracellular domains to change the ligand binding affinity [59]. It is currently unclear whether DAP-kinase acts directly or indirectly on integrin cytoplasmic domains. Recent study revealed that the interaction of cytoplasmic protein talin with the integrin $\beta$ subunit cytoplasmic domain represents a common downstream event of many signaling pathways that control integrin activation [60]. Whether DAP-kinase affects talin binding to integrin $\beta$ tail thus becomes an important question to be addressed.

\section{Perspectives}

Ten years after the identification of DAP-kinase, evidence has now emerged that this cytoskeleton localized kinase promotes cell death partly through its action on cytoskeleton and cytoskeleton-associated molecules, such as integrins. In addition to typical caspase-dependent death (apoptosis), DAP-kinase can also trigger autophagic death under certain circumstances. Although MLC is the only DAP-kinase in vivo substrate identified to date, DAP-kinase elicits distinct biological effects compared with other kinases that phosphorylate MLC, such as MLCK and Rho kinase. Such differences could be resulted from their differences in interacting partners, subcellular localizations and ability to phosphorylate other cellular substrates. As all of the biological effects of DAP-kinase characterized to 
date require its catalytic activity, identification of other in vivo substrates of DAP-kinase would be important to fully understand its functional mechanism. In addition, the capabilities of DAP-kinase to phosphorylate MLC and modulate integrin activation imply that this kinase may participate in other, death-unrelated biological functions, such as cell migration, tumor invasion and cytokinesis. The possibility of DAP-kinase to affect cell migration is particularly intriguing, as downregulation of this kinase displays a tight correlation with tumor metastasis. Finally, the tumor suppressive activity of DAP-kinase suggests that targeted activation of this kinase may confer a potential therapeutic opportunity for various types of cancer. Several strategies for specifically activating this kinase have been discussed [7], and future studies will aim at testing their efficacies.

\section{Acknowledgements}

R.H.C. is supported by NSC Frontier Grant NSC94-2321-B-002-013 and Academia Sinica Thematic Project AS-95-TP-B02.

\section{References}

1. Cohen O. and Kimchi A., DAP-kinase: from functional gene cloning to establishment of its role in apoptosis and cancer. Cell Death Differ. 8: 6-15, 2001.

2. Kogel D., Prehn J.H. and Scheidtmann K.H., The DAP kinase family of pro-apoptotic proteins: novel players in the apoptotic game. Bioessays 23: 352-358, 2001.

3. Deiss L.P., Feinstein E., Berissi H., Cohen O. and Kimchi A., Identification of a novel serine/threonine kinase and a novel $15-\mathrm{kD}$ protein as potential mediators of the gamma interferon-induced cell death. Genes Dev. 9: 15-30, 1995.

4. Cohen O., Feinstein E. and Kimchi A., DAP-kinase is a $\mathrm{Ca} 2+/$ calmodulin-dependent, cytoskeletal-associated protein kinase, with cell death-inducing functions that depend on its catalytic activity. EMBO J. 16: 998-1008, 1997.

5. Shohat G., Spivak-Kroizman T., Cohen O., Bialik S., Shani G., Berrisi H., Eisenstein M. and Kimchi A., The proapoptotic function of death-associated protein kinase is controlled by a unique inhibitory autophosphorylationbased mechanism. J. Biol. Chem. 276: 47460-47467, 2001.

6. Chen C.H., Wang W.J., Kuo J.C., Tsai H.C., Lin J.R., Chang Z.F. and Chen R.H., Bidirectional signals transduced by DAPK-ERK interaction promote the apoptotic effect of DAPK. EMBO J. 24: 294-304, 2005.

7. Bialik S. and Kimchi A., DAP-kinase as a target for drug design in cancer and diseases associated with accelerated cell death. Semin. Cancer Biol. 14: 283-294, 2004.
8. Raveh T. and Kimchi A., DAP kinase - a proapoptotic gene that functions as a tumor suppressor. Exp. Cell Res. 264: 185-192, 2001.

9. Kissil J.L., Feinstein E., Cohen O., Jones P.A., Tsai Y.C., Knowles M.A., Eydmann M.E. and Kimchi A., DAPkinase loss of expression in various carcinoma and B-cell lymphoma cell lines: possible implications for role as tumor suppressor gene. Oncogene 15: 403-407, 1997.

10. Katzenellenbogen R.A., Baylin S.B. and Herman J.G., Hypermethylation of the DAP-kinase $\mathrm{CpG}$ island is a common alteration in B-cell malignancies. Blood 93: 43474353, 1999.

11. Nakatsuka S., Takakuwa T., Tomita Y., Hoshida Y., Nishiu M., Yamaguchi M., Nishii K., Yang W.I. and Aozasa K., Hypermethylation of death-associated protein (DAP) kinase $\mathrm{CpG}$ island is frequent not only in B-cell but also in $\mathrm{T}$ - and natural killer (NK)/T-cell malignancies. Cancer Sci. 94: 87-91, 2003.

12. Kwong J., Lo K.W., To K.F., Teo P.M., Johnson P.J. and Huang D.P., Promoter hypermethylation of multiple genes in nasopharyngeal carcinoma. Clin. Cancer Res. 8: 131-137, 2002.

13. Wong T.S., Chang H.W., Tang K.C., Wei W.I., Kwong D.L., Sham J.S., Yuen A.P. and Kwong Y.L., High frequency of promoter hypermethylation of the deathassociated protein-kinase gene in nasopharyngeal carcinoma and its detection in the peripheral blood of patients. Clin. Cancer Res. 8: 433-437, 2002.

14. Kim D.H., Nelson H.H., Wiencke J.K., Christiani D.C., Wain J.C., Mark E.J. and Kelsey K.T., Promoter methylation of DAP-kinase: association with advanced stage in non-small cell lung cancer. Oncogene 20: 1765-1770, 2001.

15. Sanchez-Cespedes M., Esteller M., Wu L., Nawroz-Danish H., Yoo G.H., Koch W.M., Jen J., Herman J.G. and Sidransky D., Gene promoter hypermethylation in tumors and serum of head and neck cancer patients. Cancer Res. 60: 892-895, 2000.

16. Hasegawa M., Nelson H.H., Peters E., Ringstrom E., Posner M. and Kelsey K.T., Patterns of gene promoter methylation in squamous cell cancer of the head and neck. Oncogene 21: 4231-4236, 2002.

17. Satoh A., Toyota M., Itoh F., Kikuchi T., Obata T., Sasaki Y., Suzuki H., Yawata A., Kusano M., Fujita M., Hosokawa M., Yanagihara K., Tokino T. and Imai K., DNA methylation and histone deacetylation associated with silencing DAP kinase gene expression in colorectal and gastric cancers. Br. J. Cancer 86: 1817-1823, 2002.

18. Yamaguchi S., Asao T., Nakamura J., Ide M. and Kuwano H., High frequency of DAP-kinase gene promoter methylation in colorectal cancer specimens and its identification in serum. Cancer Lett. 194: 99-105, 2003.

19. Kang G.H., Shim Y.H., Jung H.Y., Kim W.H., Ro J.Y. and Rhyu M.G., CpG island methylation in premalignant stages of gastric carcinoma. Cancer Res. 61: 2847-2851, 2001 .

20. Matsumoto H., Nagao M., Ogawa S., Kanehiro H., Hisanaga M., Ko S., Ikeda N., Fujii H., Koyama F., Mukogawa T. and Nakajima Y., Prognostic significance of death-associated protein-kinase expression in hepatocellular carcinomas. Anticancer Res. 23: 1333-1341, 2003.

21. Ng M.H., To K.W., Lo K.W., Chan S., Tsang K.S., Cheng S.H. and $\mathrm{Ng} \mathrm{H.K.,} \mathrm{Frequent} \mathrm{death-associated} \mathrm{protein}$ kinase promoter hypermethylation in multiple myeloma. Clin. Cancer Res. 7: 1724-1729, 2001. 
22. Simpson D.J., Clayton R.N. and Farrell W.E., Preferential loss of Death Associated Protein kinase expression in invasive pituitary tumours is associated with either $\mathrm{CpG}$ island methylation or homozygous deletion. Oncogene 21: 1217-1224, 2002.

23. Tada Y., Wada M., Taguchi K., Mochida Y., Kinugawa N., Tsuneyoshi M., Naito S. and Kuwano M., The association of death-associated protein kinase hypermethylation with early recurrence in superficial bladder cancers. Cancer Res. 62: 4048-4053, 2002.

24. Gonzalez-Gomez P., Bello M.J., Arjona D., Lomas J., Alonso M.E., De Campos J.M., Vaquero J., Isla A., Gutierrez M. and Rey J.A., Promoter hypermethylation of multiple genes in astrocytic gliomas. Int. J. Oncol. 22: 601608, 2003.

25. Narayan G., Arias-Pulido H., Koul S., Vargas H., Zhang F.F., Villella J., Schneider A., Terry M.B., Mansukhani M. and Murty V.V., Frequent promoter methylation of CDH1, DAPK, RARB, and HIC1 genes in carcinoma of cervix uteri: its relationship to clinical outcome. Mol. Cancer 2: 24, 2003

26. Chan A.W., Chan M.W., Lee T.L., Ng E.K., Leung W.K., Lau J.Y., Tong J.H., Chan F.K. and To K.F., Promoter hypermethylation of Death-associated protein-kinase gene associated with advance stage gastric cancer. Oncol. Rep. 13: 937-941, 2005

27. Russo A.L., Thiagalingam A., Pan H., Califano J., Cheng K.H., Ponte J.F., Chinnappan D., Nemani P., Sidransky D. and Thiagalingam S., Differential DNA hypermethylation of critical genes mediates the stage-specific tobacco smokeinduced neoplastic progression of lung cancer. Clin. Cancer Res. 11: 2466-2470, 2005

28. Inbal B., Cohen O., Polak-Charcon S., Kopolovic J., Vadai E., Eisenbach L. and Kimchi A., DAP kinase links the control of apoptosis to metastasis. Nature 390: 180-184, 1997.

29. Raveh T., Droguett G., Horwitz M.S., DePinho R.A. and Kimchi A., DAP kinase activates a p19ARF/p53-mediated apoptotic checkpoint to suppress oncogenic transformation. Nat. Cell Biol. 3: 1-7, 2001.

30. Jang C.W., Chen C.H., Chen C.C., Chen J.Y., Su Y.H. and Chen R.H., TGF-beta induces apoptosis through Smadmediated expression of DAP- kinase. Nat. Cell Biol. 4: 5158, 2002.

31. Cohen O., Inbal B., Kissil J.L., Raveh T., Berissi H., Spivak-Kroizaman T., Feinstein E. and Kimchi A., DAPkinase participates in TNF-alpha- and Fas-induced apoptosis and its function requires the death domain. J. Cell Biol. 146: 141-148, 1999

32. Tang X., Wu W., Sun S.Y., Wistuba I.I., Hong W.K. and Mao L., Hypermethylation of the death-associated protein kinase promoter attenuates the sensitivity to TRAILinduced apoptosis in human non-small cell lung cancer cells. Mol. Cancer Res. 2: 685-691, 2004.

33. Yamamoto M., Takahashi H., Nakamura T., Hioki T., Nagayama S., Ooashi N., Sun X., Ishii T., Kudo Y., Nakajima-Iijima S., Kimchi A. and Uchino S., Developmental changes in distribution of death-associated protein kinase mRNAs. J. Neurosci. Res. 58: 674-683, 1999.

34. Pelled D., Raveh T., Riebeling C., Fridkin M., Berissi H., Futerman A.H. and Kimchi A., Death-associated protein (DAP) kinase plays a central role in ceramide-induced apoptosis in cultured hippocampal neurons. J. Biol. Chem. 277: 1957-1961, 2002.
35. Yamamoto M., Hioki T., Ishii T., Nakajima-Iijima S. and Uchino S., DAP kinase activity is critical for $\mathrm{C}(2)$ ceramide-induced apoptosis in PC12 cells. Eur. J. Biochem. 269: 139-147, 2002.

36. Henshall D.C., Araki T., Schindler C.K., Shinoda S., Lan J.Q. and Simon R.P., Expression of death-associated protein kinase and recruitment to the tumor necrosis factor signaling pathway following brief seizures. J. Neurochem. 86: 1260-1270, 2003.

37. Schumacher A.M., Velentza A.V., Watterson D.M. and Wainwright M.S., DAPK catalytic activity in the hippocampus increases during the recovery phase in an animal model of brain hypoxic-ischemic injury. Biochim. Biophys. Acta 1600: 128-137, 2002.

38. Shamloo M., Soriano L., Wieloch T., Nikolich K., Urfer R. and Oksenberg D., Death-associated protein kinase is activated by dephosphorylation in response to cerebral ischemia. J. Biol. Chem. 280: 42290-42299, 2005.

39. Llambi F., Causeret F., Bloch-Gallego E. and Mehlen P., Netrin-1 acts as a survival factor via its receptors UNC5H and DCC. EMBO J. 20: 2715-2722, 2001.

40. Llambi F., Lourenco F.C., Gozuacik D., Guix C., Pays L., Del Rio G., Kimchi A. and Mehlen P., The dependence receptor UNC5H2 mediates apoptosis through DAPkinase. EMBO J. 24: 1192-1201, 2005.

41. Klionsky D.J. and Emr S.D., Autophagy as a regulated pathway of cellular degradation. Science 290: 1717-1721, 2000 .

42. Meijer A.J. and Codogno P., Regulation and role of autophagy in mammalian cells. Int. J. Biochem. Cell Biol. 36: 2445-2462, 2004.

43. Inbal B., Bialik S., Sabanay I., Shani G. and Kimchi A., DAP kinase and DRP-1 mediate membrane blebbing and the formation of autophagic vesicles during programmed cell death. J. Cell Biol. 157: 455-468, 2002.

44. Yu L., Alva A., Su H., Dutt P., Freundt E., Welsh S., Baehrecke E.H. and Lenardo M.J., Regulation of an ATG7-beclin 1 program of autophagic cell death by caspase-8. Science 304: 1500-1502, 2004.

45. Shimizu S., Kanaseki T., Mizushima N., Mizuta T., Arakawa-Kobayashi S., Thompson C.B. and Tsujimoto Y., Role of Bcl-2 family proteins in a non-apoptotic programmed cell death dependent on autophagy genes. Nat. Cell Biol. 6: 1221-1228, 2004.

46. Bialik S., Bresnick A.R. and Kimchi A., DAP-kinasemediated morphological changes are localization dependent and involve myosin-II phosphorylation. Cell Death Differ. 11: 631-644, 2004.

47. Kuo J.-C., Lin J.-R., Staddon J.M., Hosoya H. and Chen R.-H., Uncoordinated regulation of stress fibers and focal adhesions by DAP-kinase. J. Cell Sci. 116: 4777-4790, 2003.

48. Frisch S.M. and Screaton R.A., Anoikis mechanisms. Curr Opin. Cell Biol. 13: 555-562, 2001.

49. Streuli C.H. and Gilmore A.P., Adhesion-mediated signaling in the regulation of mammary epithelial cell survival. J. Mammary Gland Biol. Neoplasia 4: 183-191, 1999.

50. Shanmugathasan M. and Jothy S., Apoptosis, anoikis and their relevance to the pathobiology of colon cancer. Pathol. Int. 50: 273-279, 2000

51. Wei L., Yang Y. and Yu Q., Tyrosine kinase-dependent, phosphatidylinositol 3'-kinase, and mitogen-activated protein kinase-independent signaling pathways prevent lung adenocarcinoma cells from anoikis. Cancer Res. 61: 24392444,2001 
52. Wang W.J., Kuo J.C., Yao C.C. and Chen R.H., DAPkinase induces apoptosis by suppressing integrin activity and disrupting matrix survival signals. J. Cell Biol. 159: 169-179, 2002.

53. Stromblad S., Becker J.C., Yebra M., Brooks P.C. and Cheresh D.A., Suppression of p53 activity and p21WAF1/ CIP1 expression by vascular cell integrin alphaVbeta3 during angiogenesis. J. Clin. Invest. 98: 426-433, 1996.

54. Ilic D., Almeida E.A., Schlaepfer D.D., Dazin P., Aizawa S. and Damsky C.H., Extracellular matrix survival signals transduced by focal adhesion kinase suppress p53-mediated apoptosis. J. Cell Biol. 143: 547-560, 1998.

55. Chipuk J.E., Kuwana T., Bouchier-Hayes L., Droin N.M., Newmeyer D.D., Schuler M. and Green D.R., Direct activation of Bax by p53 mediates mitochondrial membrane permeabilization and apoptosis. Science 303: 10101014, 2004.
56. Leu J.I., Dumont P., Hafey M., Murphy M.E. and George D.L., Mitochondrial p53 activates Bak and causes disruption of a Bak-Mcll complex. Nat. Cell Biol. 6: 443-450, 2004.

57. Slee E.A., O'Connor D.J. and Lu X., To die or not to die: how does p53 decide?. Oncogene 23: 2809-2818, 2004.

58. Martin S.S. and Vuori K., Regulation of Bcl-2 proteins during anoikis and amorphosis. Biochim. Biophys. Acta 1692: 145-157, 2004.

59. Liddington R.C. and Ginsberg M.H., Integrin activation takes shape. J. Cell Biol. 158: 833-839, 2002.

60. Tadokoro S., Shattil S.J., Eto K., Tai V., Liddington R.C., de Pereda J.M., Ginsberg M.H. and Calderwood D.A., Talin binding to integrin beta tails: a final common step in integrin activation. Science 302: 103-106, 2003. 